\title{
Uso de las ciencias del comportamiento en políticas públicas: perspectivas desde la pandemia
}

Use of behavioral sciences in public policies: perspectives from the pandemic

\author{
Recepción del artículo: 06-07-2021 | Aceptación del artículo: 26-01-2022
}

\begin{abstract}
Abrevaya, Sofia ${ }^{1,2}$
abrevayasofia@gmail.com

iD https://orcid.org/0000-0003-4091-9962

Putrino, Natalia Inés $\mathbf{s}^{2,3,4}$

nataliaines.putrino@gmail.com

$\underline{\text { https://orcid.org/0000-0001-8205-9070 }}$

Tomio, Ailin 5

ailintomio@gmail.com

https://orcid.org/0000-0002-5809-2986

Bibbo, Diana ${ }^{5}$

dianabibbo@gmail.com

Dhttps://orcid.org/0000-0002-4998-0826

Yoris, Adrián Ezequiel1,2

aeyoris@gmail.com
\end{abstract}

Dhttps://orcid.org/0000-0003-4208-0603

Ramenzoni, Verónica Claudia ${ }^{2,6}$ vramenzoni@gmail.com

https://orcid.org/0000-0003-3003-5012

${ }^{1}$ Instituto de Neurociencia Cognitiva y Traslacional (Consejo Nacional de Investigaciones Científicas s Técnicas - Fundación INECO - Universidad Favaloro)

${ }^{2}$ Consejo Nacional de Investigaciones Científicas y Técnicas (CONICET) ${ }^{3}$ Instituto de Ciencias para la Familia, Universidad Austral ${ }^{4}$ Facultad de Psicología, Universidad de Buenos Aires ${ }^{5}$ Facultad de Ciencias Humanas y de la Conducta Universidad Favaloro ${ }^{6}$ Centro de Investigaciones en Psicología Psicopedagogía, Universidad Católica Argentin

Para referenciar este artículo:

Abrevaya, S., Putrino, N. I., Tomio, A., Bibbo, D., Yoris, A. y Ramenzoni, V. C. (2022). Uso de las ciencias de comportamiento en políticas públicas: perspectivas desde la pandemia. ConCiencia EPG, 7(1), 101-123 https://doi.org/10.32654/CONCIENCIAEPG.7-1.7

Autora corresponsal: Sofía Abrevaya abrevayasofia@gmail.com. Buenos Aires, Argentina

\section{Resumen}

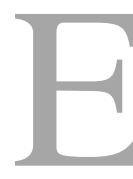

xiste un creciente interés en aplicar los hallazgos de las ciencias del comportamiento a los desafíos de formulación de políticas públicas debido a su bajo costo y alto impacto en el corto plazo. Experiencias previas han mostrado los beneficios de utilizar las ciencias del comportamiento en políticas de salud tales como lograr adherencia a tratamientos crónicos. En el contexto de la pandemia del COVID-19, se ha reforzado la importancia de estas medidas para contener la expansión de la enfermedad y promover la vacunación de la población. El objetivo de este trabajo es repasar la evidencia de las ciencias del comportamiento en el control de la pandemia. Asimismo, se realiza una breve discusión sobre cómo aplicarlo a futuro de forma sistemática en las políticas públicas, realizando un recorrido por experiencias realizadas en otros países, haciendo foco en la promoción del proceso vacunatorio.

Palabras Clave: ciencias del comportamiento, behavioral insights, pandemia, políticas públicas. 
Abstract

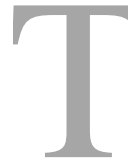

here is a growing interest in applying behavioral sciences' findings to public policy formulation, due to their low cost and high impact in the short term. Previous experiences have shown the benefit of applying behavioral sciences to design health policies, such as achieving adherence to chronic treatments. In the context of the COVID-19 pandemic, the importance of these measures to contain the spread of the disease and promote vaccination of the population has been reinforced. The objective of this work is to review the evidence of the behavioral sciences in the control of the pandemic. Likewise, a brief discussion is held on how to apply it in the future in a systematic way in public policies, making a tour of experiences in other countries, focusing on the promotion of the vaccination process.

Key Words: behavioral science, behavioral insights, pandemic, public policy.

\section{Introducción}

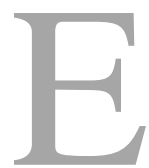

n la última década ha surgido un creciente interés en aplicar conocimientos de las ciencias del comportamiento a los desafíos de la formulación y el diseño de políticas públicas (Galizzi, 2017). La Organización para la Cooperación y el Desarrollo Económicos (OCDE) ha estado a la vanguardia de la documentación e investigación acerca del uso de los principios conductuales en los programas públicos en general y en los campos de políticas como la protección del consumidor, el medio ambiente, la educación, la integridad del sector público, la obesidad, la política regulatoria y los impuestos (OECD, 2017a, 2019). Estas intervenciones comportamentales se definen generalmente como una intervención política que está directamente inspirada y diseñada en los principios de investigación de la conducta humana. El objetivo clave de estas intervenciones es difundir los aprendizajes de las ciencias del comportamiento provenientes de la investigación en la comunidad de profesionales de política pública (Behavioural Insights Team, 2014).

La aplicación del conocimiento de las ciencias sociales y del comportamiento en las políticas públicas se conoce como Behavioral Insights (BI), una disciplina que se basa en la Economía del Comportamiento y sus aplicaciones a las políticas públicas (Calvo-González \& Zoratto, 2017). La Economía del Comportamiento es una disciplina cuyos inicios se asocian al trabajo de Daniel Kahneman y Amos Tversky, en el que intentan comprender cómo los comportamientos se desvían de lo que esperaría el modelo racional de las teorías económicas clásicas (Kahneman \& Egan, 2011; Tversky \& Kahneman, 1974). El estudio de los procesos de toma de decisiones es vital para comprender por qué las personas eligen comportamientos que pueden ser contraproducentes, en lugar de comportamientos que son más beneficiosos para su economía, salud o medio ambiente. BI es un nuevo enfoque 
que puede colaborar con los gobiernos y organizaciones a reducir sus cargas regulatorias sobre las empresas y la sociedad, al mismo tiempo que ayuda a lograr sus objetivos de políticas de una manera económica y eficaz (Behavioural Insights Team, 2019; Hallsworth \& Kirkman, 2020).

Los ensayos de BI generalmente implican el uso de "empujones" (Nudges); intervenciones que preservan la capacidad de elección y que guían indirectamente los comportamientos en direcciones específicas (Sunstein et al., 2017). Los empujones incluyen desde cambios en la información a advertencias, recordatorios, creación de normas sociales, o reglas predeterminadas (Thaler \& Sunstein, 2009). Existen distintos tipos de estudios en BI (Lourenço et al., 2016) pero se pueden identificar principalmente tres tipos: iniciativas probadas conductualmente (es decir, probadas por primera vez), informadas conductualmente (es decir, diseñadas sobre evidencia conductual existente, lo que implica que la evidencia es suficiente para aplicar la intervención sin probarla) y estudios conductuales alineados (es decir, intervenciones no probadas que, a posteriori, se encuentra que están en línea con la evidencia conductual).

Es así, que las intervenciones sobre la conducta pueden clasificarse según el mecanismo central que está siendo intervenido para su ejecución. Uno de los tipos de intervenciones más comunes es el uso de incentivos para reforzar el cambio de comportamiento (por ej., una reducción de la tarifa de un impuesto, si el sujeto paga en tiempo y forma), una herramienta política tradicional que muchos gobiernos han utilizado para promover determinados comportamientos, como el hábito de fumar, u otros hábitos no saludables (Cahill \& Perera, 2011; John et al., 2011; Surrey, 1970). La investigación sobre el tipo y los modos de implementación de los incentivos proporciona información adicional que puede aumentar su efectividad y reducir sus costos. Dos de las formas más populares de incentivos que utilizan los gobiernos son los incentivos fiscales y las transferencias monetarias condicionadas (Fiszbein \& Schady, 2009).

También puede modificarse el formato en que es presentada la información, teniendo en cuenta su prominencia. La forma en que se transmite la información puede cambiar las respuestas de las personas a los estímulos. Hacer que una pieza de información sobresalga implica dirigir la atención de las personas hacia ella, lo que a su vez maximiza las posibilidades de que respondan a ella (Behavioural Insights Team, 2014).

Por otro lado, el compromiso es una promesa de comportarse de cierta manera o actuar hacia un objetivo específico (Ashraf, 2013; Mayer et al., 2015). Un ejemplo de compromiso es depositar dinero en una cuenta y no poder acceder a ella hasta haber cumplido con el objetivo propuesto o si no se pierde lo invertido. Esto impulsa la aversión a las pérdidas (la tendencia de las personas a preferir evitar pérdidas a adquirir ganancias) y de esa manera cumplir con el objetivo. Asimismo, se puede estimular a que las personas 
expresen públicamente sus intenciones que genera un aumento en la necesidad de ser coherente (Mayer et al., 2015). Siempre que las intenciones contradicen el comportamiento, las personas experimentan disonancia cognitiva (Festinger, 1962). Por esta razón, expresar un compromiso públicamente aumenta la probabilidad de que las personas se sientan estimuladas a honrarlo.

Otro modo de intervenir sobre una situación es a través de la simplificación. A veces, los pequeños cambios que reducen la fricción en un proceso de toma de decisiones pueden tener un gran impacto (Behavioural Insights Team, 2010). Los mensajes y los procesos se pueden simplificar para aumentar su comprensión para los destinatarios (Bettinger et al., 2012).

Asimismo, un método que ha crecido con el desarrollo de las redes sociales es el de influencia social. Los humanos somos seres sociales fuertemente influenciados por lo que otros hacen y dicen (Hallsworth et al., 2016). El Behavioural Insights Team (BIT) (Behavioural Insights Team, 2010), utilizó esta suposición para aumentar las tasas de donación (Behavioural Insights Team, 2013), recaudación de impuestos (Larkin et al., 2018), y donaciones caritativas (Sanders, 2017), entre otras cuestiones. La literatura académica sobre cómo las personas se influencian entre sí está en aumento (Sanders, 2017) y consideramos que es una categoría relevante para este estudio.

Por último, la gamificación ha sido definida (Hallsworth et al., 2016) como el uso de juegos para involucrar a los usuarios en el logro de objetivos. Esto se refiere a plataformas como aplicaciones de teléfonos móviles, refiriéndose a juegos digitales. No estamos lejos de que la mayoría de los teléfonos sean inteligentes, lo que significa que las personas poseen un teléfono que incorpora servicios de GPS, acelerómetros, sensores externos, etc. Las recompensas pueden ser virtuales (como la competencia social) o tangibles si se realizan en un entorno de programa de incentivos (por ejemplo, el plan de incentivos para viajeros de Singapur recompensa a los viajeros por pasar de los trenes de hora pico a los de menor actividad).

También la gamificación ha tenido una buena repercusión para promover la adherencia a prácticas médicas, por ejemplo, un medidor de glucosa (DIDGET) en niños, refuerza positivamente los hábitos constantes de análisis de glucosa en sangre al otorgar puntos que los niños pueden usar para desbloquear nuevos niveles de juego y personalizar su experiencia de juego (Klingensmith et al., 2013). Estos juegos con propósito pretenden acciones tales como mejorar la salud y promover o mantener hábitos saludables. Esto se logra a partir de la fusión del diseño de software y la mecánica del juego con la teoría de la salud pública y los conocimientos del comportamiento. Por su propia naturaleza, los juegos están diseñados para motivar el comportamiento del usuario. Algunas de las características utilizadas para alcanzar esto están relacionadas con conocimientos de la Economía del Comportamiento. Por ejemplo, el conocimiento de las tendencias de las personas para evitar pérdidas 
(aversión a las pérdidas) y para valorar irracionalmente las cosas que tienen sobre las cosas que no tienen (efecto de dotación), se aplica al proporcionar recompensas condicionales (como puntos y premios) que corren el riesgo de perderse si los jugadores no regresan con frecuencia a jugar (King et al., 2013).

Durante la última década, gobiernos de diferentes países han utilizado BI para mejorar políticas en diferentes áreas como el de la salud, ahorro para la jubilación, pago de impuestos, medio ambiente, matrícula escolar, etc. (Halpern, 2015; OECD, 2017b; Sunstein et al., 2017). Se ha demostrado la eficacia de utilizar de utilizar las ciencias del comportamiento en políticas públicas en otros gobiernos nacionales (Galizzi, 2017; Vlaev et al., 2016). Si bien más de 150 países en todo el mundo están utilizando estas herramientas para influir en el comportamiento, en países de Latinoamérica las estrategias de conocimiento del comportamiento son menos conocidas y, por lo tanto, se han utilizado poco (Sunstein et al., 2017). En Latinoamérica los estudios de BI son escasos y los publicados tienden a centrarse en temáticas tales como la conservación del agua (Calvo-González \& Zoratto, 2017; Datta et al., 2015), el cumplimiento tributario (Brockmeyer et al., 2016; CalvoGonzález \& Zoratto, 2017; Castro \& Scartascini, 2015; Kettle et al., 2016), las transferencias monetarias condicionadas (Calvo-González \& Zoratto, 2017), y el comportamiento materno (García Prado, 2016).

Sin embargo, estas no han sido incorporadas en el sistema gubernamental de la Argentina. En particular, en el contexto de la pandemia se ha reforzado la importancia de estas para contener el esparcimiento de la enfermedad (Lunn, Belton, et al., 2020; Van Bavel et al., 2020; West et al., 2020). El objetivo de este artículo es repasar la evidencia de las ciencias del comportamiento en el control de la pandemia, además de resumir y discutir las recomendaciones internacionales realizadas sobre cómo utilizarlas. Asimismo, se realiza una breve discusión sobre cómo aplicarlo a futuro de forma sistemática en las políticas públicas, realizando un recorrido por experiencias realizadas en otros países, haciendo foco en la promoción del proceso vacunatorio. En este marco, hemos analizado publicaciones que presentan las estrategias conductuales como su principal intervención, pero también estudios que utilizaron las iniciativas conductuales como una forma de mejorar su estrategia principal.

\section{Ciencias del comportamiento en la pandemia del COVID-19}

En vistas de una crisis mundial derivada de una pandemia sanitaria, profesionales e investigadores de las ciencias del comportamiento se preguntaron qué aportes puede y tiene el campo para ayudar en la pandemia. El axioma principal se sostiene en que el comportamiento humano forma parte del núcleo de la pandemia: su origen, su dispersión, y su mantenimiento. Los aportes pueden dividirse de dos maneras: desde la investigación de los factores que generan conductas de impacto negativo para la pandemia (Betsch et al., 2020; World Health Organization, 2020d) y a la 
implementación directa de tácticas comportamentales en políticas públicas basadas en experiencias previas (Finset et al., 2020; Martínez Villareal et al., 2020).

Un estudio reciente probó estrategias de comunicación para promover el distanciamiento a través de un experimento online encargado por el Departamento de Salud de Irlanda (Lunn et al., 2020). Un grupo de control vio un cartel informativo actual y dos grupos de tratamiento vieron carteles similares con mensajes que destacaban: (i) el riesgo de transmisión a personas identificables vulnerables al COVID-19; (ii) la naturaleza exponencial de la transmisión. Posteriormente midieron los juicios de los comportamientos previamente identificados por los grupos focales como "marginales" (lo que significaba que las personas no estaban seguras de si eran aconsejables, como encontrarse con otros al aire libre o visitar a los padres). Los hallazgos sugieren que la idea de infectar a personas vulnerables o un gran número de personas puede motivar el distanciamiento social.

En este mismo sentido, el autoaislamiento y el distanciamiento social son dos caras de la misma moneda. Un experimento en línea que tuvo lugar en Irlanda probó ayudas para la toma de decisiones basadas en el comportamiento para apoyar el autoaislamiento (Lunn, et al., 2020). El experimento tuvo tres etapas que probaron intervenciones diseñadas para ayudar a las personas a: (i) decidir si necesitan aislarse a sí mismas; (ii) tener confianza en su capacidad para aislarse a sí mismos si es necesario; y (iii) administrar un hogar en el que un individuo necesita aislarse por sí mismo. En relación con los consejos de salud pública predominantes, mostrar árboles de decisiones mejoró las decisiones de los participantes sobre cuándo era necesario el autoaislamiento, aunque sistemáticamente subestimaron la necesidad de auto aislarse en presencia de síntomas menos comunes de COVID-19 como dolor de garganta $\mathrm{y}$ fatiga. $\mathrm{La}$ interacción con una herramienta de planificación en línea aumentó la confianza para afrontar el autoaislamiento entre los adultos menores de 40 años. La presentación de consejos en forma de infografías mejoró el recuerdo y la comprensión de cómo manejar el autoaislamiento. El estudio demuestra cómo las políticas de salud pública pueden beneficiarse de las pruebas previas del resultado de las intervenciones.

Una herramienta utilizada en ciencias del comportamiento es el encuadre prosocial (Rudd et al., 2014) que implica enmarcar metas en un contexto prosocial, como hacer felices a los demás o salvar el medio ambiente. Un grupo de investigadores midió la influencia de tres tipos de mensajería en las intenciones de prevención del coronavirus entre los estadounidenses de Amazon Mechanical Turk (Jordan et al., 2020). Todos los tipos presentaron información de COVID-19 idéntica, pero enfatizaron en los beneficios personales, públicos o tanto personales como públicos de las conductas de prevención. En otros estudios realizados al comienzo de la pandemia (entre el 14 y 16 de marzo, momento en el que en Estados Unidos habían menos de 2000 casos confirmados) encontraron apoyo para el encuadre prosocial: el tratamiento público 
fue más efectivo que el tratamiento personal, y no menos efectivo que el trato personal y público. En estudios posteriores (realizados del 17 al 30 de abril, cuando habían más de 500.000 casos confirmados en EE. UU.), se halló que tres tratamientos fueron igualmente efectivos. Además, en ambos conjuntos de estudios, la amenaza pública percibida del Coronavirus predijo las intenciones de prevención con más fuerza que la amenaza personal percibida.

Debido a sus beneficios, se ha propuesto como herramienta económica y eficaz el uso de los medios digitales para transmitir información a la población (García Zaballos et al., 2020; Ienca \& Vayena, 2020; Whitelaw et al., 2020). Un estudio evaluó los comportamientos preventivos contra la contracción de COVID-19 y probó vías de mediación que vinculan cuatro tipos de consumo de medios digitales: redes sociales, aplicaciones de redes sociales móviles (MSN), medios de noticias en línea y servicios de transmisión en vivo de redes sociales (SLSS) con comportamientos preventivos mediados por la preocupación (Liu, 2020). Los resultados de una encuesta en línea revelaron que la gran mayoría (> 90 por ciento) de los encuestados había practicado muy a menudo o siempre comportamientos preventivos contra el COVID-19, como lavarse las manos con más frecuencia con agua y jabón, mantenerse alejado de lugares llenos de gente y usar máscaras faciales en público. Además, la búsqueda de información relacionada con COVID-19 en MSN, SLSS y medios de comunicación en línea se asoció directamente con conductas preventivas. Asimismo, un estudio en Zimbabue mostró como la transmisión de información en medios digitales y confiables pueden modificar el comportamiento de la población. Ellos distribuyeron mensajes dirigidos a la desinformación sobre el virus a través de WhatsApp. La exposición a estos mensajes redujo un $30 \%$ el no cumplimiento de distanciamiento social (Bowles et al., 2020).

Un grupo de investigación estudió las creencias de las personas sobre las probabilidades de infección y la capacidad para practicar el distanciamiento social en el Reino Unido, EE. UU. y Alemania en marzo de 2020 y encontró que las personas muestran un sesgo de optimismo, es decir que estiman la probabilidad de infectarse con el virus y de infectar otros, si se infectan a sí mismos, son más bajos para ellos que para alguien similar a ellos (Kuper-Smith et al., 2020).

Por otro lado, como se menciona previamente, varios grupos de trabajo tanto del ámbito académico como aplicado de las ciencias del comportamiento realizaron revisiones de las experiencias e investigaciones previas en el campo de la aplicación de BI en políticas públicas para realizar propuestas de aplicación en el contexto actual. Además de las medidas de aislamiento y distanciamiento social, incluídos los edictos para quedarse en casa, existe una necesidad urgente de intervenciones efectivas para aumentar la adherencia a los comportamientos que las personas en las comunidades pueden adoptar para protegerse a sí mismos y a los demás. 
Las recomendaciones preliminares para implementar comportamientos que limiten la transmisión del virus incluyen (West et al., 2020): educación (promover la comprensión de los beneficios de los comportamientos y cómo y cuándo implementarlos de manera efectiva); persuasión (promover la preocupación y el compromiso activo en lugar de la ansiedad y la evitación defensiva), incentivar (uso de recompensas sociales para lograr que las personas se sientan positivas por haberse involucrado en el comportamiento); coerción (contingencias claramente establecidas y explicadas, la aplicación debe ser inmediata y debe centrarse en el comportamiento en lugar de en la persona); entrenamiento (demostrando el comportamiento en su totalidad y componente por componente); restricción (establecer reglas sociales claras y específicas en torno a los comportamientos); reestructuración ambiental (incluir señales ambientales que promuevan el comportamiento); modelado (mostrar a las personas con las que el grupo objetivo se identifica y que actúan en el comportamiento); y habilitación (intervenciones para mejorar la capacidad tanto para extinguir como para desarrollar hábitos y crear reglas "si-entonces").

Dentro de los mensajes predominantes del contexto de pandemia, se delimitan cinco falsas dicotomías relacionadas con COVID-19: 1) salud y vidas versus economía y medios de vida, 2) bloqueo indefinido versus reapertura ilimitada, 3) síndrome respiratorio agudo severo sintomático versus asintomático infección por coronavirus 2 (SARS-CoV-2), 4) transmisión por gotitas frente a aerosol del SARS-CoV-2, y 5) máscaras para todos frente a no usarlas (Escandón et al., 2020). Los efectos en la salud física y mental y el alto impacto económico de COVID-19 no pueden ser ignorados. Por ejemplo, los gobiernos pueden considerar la posibilidad de congelar los ingresos y pagos básicos universales de los alquileres y préstamos para todas las personas, y las licencias pagadas para los trabajadores infectados y expuestos. También es necesario garantizar las cadenas de suministro de alimentos, mantener los servicios de atención médica ambulatorios esenciales al rendimiento habitual, disminuir el desempleo, adaptar las empresas y minimizar las quiebras. La OCDE ha recomendado subir los impuestos verdes y a la propiedad para financiar la recuperación (OECD, 2020).

Por otro lado, dado que la eliminación del riesgo no es factible en medio de esta pandemia, el paso correcto es defender una estrategia sostenible como la reducción de daños, ya que arrojar vergüenza y estigma a las personas que violan las medidas preventivas probablemente refuerce negativamente los comportamientos de riesgo en lugar de reducirlos, y debe evitarse. Se recomiendan las actividades al aire libre de menor riesgo, los turnos escalonados, el teletrabajo y el rediseño de los lugares de vida y de trabajo para evitar el hacinamiento, optimizar la ventilación para abordar la fatiga de la cuarentena y aliviar el daño económico al tiempo que se protege la salud pública. Por otro lado, se debe tener más cuidado al estandarizar las definiciones de los síntomas a fin de evitar los errores de clasificación errónea y comprender el verdadero papel del espectro de presentación de COVID-19 en el 
impulso de la pandemia. En este sentido, si bien la transmisión del SARS-CoV-2 no se puede separar en la dicotomía de gotitas frente a aerosoles, adoptar una posición de confrontación contra las autoridades sanitarias como la Organización Mundial de la Salud (OMS) y el "alarmismo por aerosoles" es decididamente inútil. Por último, en consonancia con una de las orientaciones de la OMS sobre máscaras (World Health Organization, 2020a), se aboga por un enfoque de enmascaramiento comunitario "inteligente" o basado en el riesgo en lugar de un enfoque de enmascaramiento universal.

Hace más de 100 años, la revista Science publicó un artículo sobre las lecciones de la pandemia de gripe (mal llamada) española (Soper, 1919). El documento argumentó que tres factores principales se interponen en el camino de la prevención: (i) las personas no aprecian los riesgos que corren, (ii) va en contra de la naturaleza humana que las personas se encierren en un aislamiento rígido como un medio para proteger a los demás, y (iii) las personas a menudo actúan inconscientemente como un peligro continuo para ellas mismas y para los demás. Un artículo actual proporciona algunas ideas del siglo pasado sobre temas relacionados en las ciencias sociales y del comportamiento que pueden ayudar a los funcionarios de salud pública a mitigar el impacto de la pandemia actual (Van Bavel et al., 2020). Incluyen propuestas tales como identificar fuentes que sean creíbles para diferentes audiencias para compartir mensajes de salud pública, y otras tácticas para información de salud pública más específica, ya que el modelado por miembros del grupo que son centrales en las redes sociales es más efectivo para promover comportamientos prosociales. Además, los mensajes deben (i) enfatizar los beneficios para el receptor, (ii) enfocarse en proteger a los demás, (iii) alinearse con los valores morales del receptor, (iv) apelar al consenso social o las normas científicas y / o (v) resaltar la perspectiva de la aprobación del grupo social que tiende a ser persuasiva. Hay un consenso general también en reemplazar el término "distanciamiento social" por "distanciamiento físico", porque permite el hecho de que la conexión social es posible incluso cuando las personas están separadas físicamente. Una revisión narrativa rápida encontró intervenciones conductuales efectivas para aumentar el lavado de manos, pero no para reducir el contacto facial (Lunn, Belton, et al., 2020).

Por último, no debe menospreciarse la importancia en particular de los aspectos psicológicos de la pandemia: desde el efecto de la cuarentena sobre la salud mental en general (Brooks et al., 2020; Garfin et al., 2020; Hossain et al., 2020; Lima et al., 2020; Usher et al., 2020), el pánico relativo a la crisis sanitaria (Kaslow et al., 2020), los afectados de forma directa por la enfermedad (Duan \& Zhu, 2020; Greenberg et al., 2020), hasta el consecuente cambio de hábitos post-pandemia (Kessler \& Wittchen, 2008). La ciencia psicológica tiene un largo historial de prevención y atención primaria de la salud mental en contexto de catástrofes o crisis, y su aporte no va solo de la mano de una perspectiva sanitaria, sino que es parte de los motivos por los cuales las conductas de la población pueden ir variando según el contexto de 
encierro. En particular se han propuestos grupos de asistentes e interventores psicológicos, mediados con el uso de tecnologías de internet (Urzúa et al., 2020). En una primera base se encuentran las comunidades, que principalmente brindan apoyo psicosocial, y poder identificar $\mathrm{y}$ ayudar a los grupos objetivo que necesitan intervención. No menos relevante es el rol preventivo de este aspecto en el contexto post-pandemia (Galea et al., 2020).

Los gobiernos y las organizaciones no gubernamentales de todo el mundo han realizado grandes esfuerzos para frenar la tasa de infección mediante la promoción o prescripción legal de comportamientos que pueden reducir la propagación del virus. Un tema que preocupa de forma creciente a los gobiernos es la circulación de información falsa o errónea que genera no solamente pánico en la población sino también el efecto contrario, de desestimar la gravedad de la enfermedad. En dos estudios con adultos estadounidenses reclutados en línea, se encontró evidencia acerca de que las personas comparten afirmaciones falsas sobre COVID-19 en parte porque simplemente no piensan lo suficiente sobre si el contenido es preciso o no al decidir qué compartir (Pennycook et al., 2020).

Otro experimento en línea con participantes en los EE. UU. y el Reino Unido, encontró que las personas sobreestiman drásticamente la infecciosidad del COVID-19 en relación con la opinión de los expertos (Akesson et al., 2020). Sin embargo, proporcionar a las personas información experta corrige parcialmente sus creencias sobre el virus.
Además, cuanto más contagiosa cree la gente que es el COVID-19, menos dispuesta está a tomar medidas de distanciamiento social, un hallazgo que denominaron "efecto fatalismo". Por último, en tres estudios de los Estados Unidos y el Reino Unido se probó la idea de que las diferentes formas de creencias conspirativas a pesar de estar correlacionadas positivamente tienen distintas implicaciones conductuales (Imhoff \& Lamberty, 2020). Mientras que las creencias de conspiración que describen la pandemia como un engaño se asociaron más fuertemente con un comportamiento reducido relacionado con la contención, las creencias de conspiración sobre fuerzas siniestras que crearon intencionalmente el virus se relacionaron con un aumento en el comportamiento de preparación egocéntrico (por ejemplo, atesorar bienes cotidianos, depender de fuentes "alternativas" de información o recursos).

\section{Aceptación de las vacunas}

Finalmente, luego de meses de incertidumbre las vacunas contra la COVID19 son hoy una realidad. Actualmente, ya se encuentran en todo el mundo (incluido Argentina) en proceso de aplicación a la población. Sin embargo, una vez que se hayan superado los retos inminentes que representan el suministro suficiente y la distribución eficaz de las vacunas y el acceso equitativo a las mismas, se necesitará una serie de estrategias bien diseñadas para fomentar su aceptación y asimilación.

La OMS remarca que teniendo en cuenta el suministro limitado a corto y medio plazo las vacunas se deben 
administrar con prioridad al personal sanitario con alto riesgo de contraer o transmitir la infección, así como a los adultos de edad avanzada (World Health Organization, 2020b). Luego, las actividades de vacunación se ampliarán a distintas poblaciones a las que no suelen llegar los programas de inmunización en cada país. Pero las investigaciones han demostrado que para fomentar la aceptación de las vacunas no basta con proporcionar información al respecto.

A su vez, el Grupo Consultivo Técnico de la OMS sobre conocimientos y ciencias del comportamiento para la salud, publicó un informe en donde se describen los factores clave a la hora de promover conductas favorables a la vacunación. Por ejemplo, uno de ellos consiste en que el proceso de vacunación debe ser rápido y fácil de modo que evite los efectos que las fricciones tradicionales del sistema de salud generan. El contexto de vacunación debe ser cómodo, seguro y familiar, para disminuir el miedo al proceso. La comunicación debe ser creíble y clara, a través de fuentes fiables. Por último, en el informe se sugiere utilizar la influencia social que provoca la conducta de miembros respetados por la sociedad (World Health Organization, 2020b).

Varios grupos influyen en la aceptación de las vacunas por COVID-19, entre ellos, los tomadores de decisiones, los coordinadores de los programas de inmunización, las organizaciones civiles y los líderes religiosos y comunitarios. Influencias sociales desfavorables pueden convertirse en una barrera muy fuerte para promover la inmunización masiva, como, por ejemplo, las creencias sobre lo que hace el grupo propio o bien lo que es aprobado o no por ese grupo (World Health Organization, 2020b). Los medios son una fuente de influencia muy potente también. Las narrativas que se distribuyen de manera masiva tienden a afectar la percepción de lo que la mayoría de las personas creen y hacen. Una revisión reciente ha encontrado que la narrativa o storytelling es una de las mejores formas para generar adherencia a la vacunación, ya que justamente los grupos antivacunas suelen ser muy eficaces con sus narrativas generando miedo en la población. También acuerdan sobre la importancia de un lenguaje acorde y emocional para la población (no enfocarse en información estadística) y la difusión de los mismos a través de personas respetadas y que inspiran confianza (Olson et al., 2020). Es por ello por lo que, en el contexto de pandemia en donde la gente se ve confinada en sus hogares y disminuye su interacción social directa, la influencia de los medios tiende a ser aún mayor, mostrando a su propio criterio cuál es la norma social (Lewandowsky et al., 2012; Rimal \& Storey, 2020).

Otro factor que influye en la reacción social hacia las vacunas es el contexto de incertidumbre desde el cual partimos. El desconocimiento general del funcionamiento del virus y el mal manejo de la crisis en gran parte de los países, alimentado por mensajes de sospecha y escepticismo en las redes y medios, promueven desconfianza a nivel poblacional. Para contrarrestar los efectos negativos de las narrativas que se oponen a las vacunas, además de promover procesos 
sin fricciones como sugiere la OMS, las campañas públicas deben orientarse a resaltar la importancia de la vacunación a nivel social tanto como individual. Atendiendo al hecho de que el riesgo a la mortalidad y morbilidad de grupos etarios jóvenes no es el mismo que los adultos mayores, es necesario destacar que la vacunación no sólo previene la enfermedad individual, sino que evita el contagio hacia terceros (Sanketh Andhavarapu, 2020).

Por otro lado, se recomienda hacer hincapié en los riesgos de la enfermedad a través de lenguaje emocional e imágenes vívidas. Las ciencias del comportamiento nos muestran que apelar a las emociones permite captar la atención y aumentar el recuerdo de información por parte de los individuos. Por lo tanto, si se busca que cierto mensaje sobresalga entre la abundancia de información existente es importante utilizar recursos que demuestren ser eficaces. Por último, en el actual contexto, donde ya comenzaron las primeras vacunaciones, mostrar individuos que atraviesan el proceso sin mayores problemas puede alentar a la sensación de seguridad a la hora de decidir si vacunarse o no.

\section{Discusión}

El uso de las ciencias sociales y del comportamiento, para informar acerca de la efectividad de distintas herramientas, para llevar a cabo diferentes políticas públicas, ha aumentado en popularidad en los últimos años (Hallsworth et al., 2016; Madrian, 2014). Ya en 2014, 51 países habían utilizado BI dentro del mismo gobierno estatal para desarrollar políticas de manera sostenida (Hallsworth et al., 2016). En Latinoamérica, la BI está comenzando a ser reconocida por los diferentes gobiernos regionales, pero su uso aún no se encuentra ampliamente extendido. En resumen, se recomienda que el conocimiento respecto al BI sea difundido con mayor fuerza en Latinoamérica con el fin de promover su aplicación e incluir los estudios actuales en este marco para hacerlos más relevantes para los gobiernos locales y las ONG que trabajan en la región.

Desafortunadamente, el uso de evidencia académica sobre el comportamiento saludable para el diseño e implementación de políticas públicas aún no representa una costumbre ampliamente extendida en Latinoamérica. Las razones de esto podrían estar relacionadas con la falta de conocimiento sobre los beneficios de las intervenciones basadas en evidencia, el asumir que las pruebas de prueba no pueden adaptarse al momento político, o incluso las falsas creencias al respecto de que puede ser demasiado costoso. Afortunadamente, esto significa que existen múltiples oportunidades en las que el BI puede mejorar el desarrollo y mostrar su valor para las políticas públicas. Este tipo de conocimiento beneficiaría no solo a los gobiernos contribuyendo a probar intervenciones de manera rentable, sino también a la comunidad académica al permitirle compartir el conocimiento específico del contexto recopilado en entornos de la vida real. Se debe aumentar la conciencia sobre la utilidad potencial del enfoque del BI. Los resultados de los estudios empíricos deben ser puestos en conocimiento de las instituciones públicas 
para lograr que éstas se involucren en una discusión más rica respecto de los temas que realmente trascienden a la hora de diseñar políticas públicas.

Algunos supuestos que están presentes con respecto a este tipo de intervenciones y que ponen en duda la legitimidad de estas como instrumento de política son: que pueden violar la toma de decisiones autónoma; su fácil implementación en las políticas públicas; y que son un medio simple y eficaz para orientar la elección individual en la dirección deseada. Un artículo reciente encontró que los empujones son una amenaza menor para la elección autónoma de lo que los críticos a veces afirman, lo que los hace éticamente más legítimos de lo que se supone a menudo (De Ridder et al., 2020). No obstante, debido a que su eficacia depende fundamentalmente de las condiciones dadas en cada contexto, su implementación no es tarea fácil, y pueden fallar (Bicchieri \& Dimant, 2019). Los estudios sobre la eficacia de los empujones deben ir más allá de la mera investigación de si estos conducen a las elecciones deseadas e incorporar medidas de agencia y autoconstitución para evaluar si son realmente capaces de aumentar la participación ciudadana. Asimismo, la comprensión de que la formulación de políticas públicas requiere procedimientos de implementación más complejos que las intervenciones conductuales regulares exige que los expertos en conducta y los formuladores de políticas públicas desarrollen nuevos métodos de colaboración. En general, entonces, se sugiere que una perspectiva integrada puede fomentar la consideración de los empujones en términos de opciones autónomas que se alinean con las preferencias individuales $\mathrm{y}$ al mismo tiempo mejorar las oportunidades de implementación al ayudar a los funcionarios de políticas públicas a superar sus dudas sobre cuándo y cómo un empujón es preferible a los públicos más convencionales.

Dadas las medidas tomadas por el Poder Ejecutivo Nacional a comienzos del 2020 respecto del Aislamiento Social Preventivo y Obligatorio (ASPO) como método eficaz para limitar el contagio y evitar el colapso de la capacidad del Sistema de Salud del país, las condiciones de prestación de servicios se han visto modificadas al pasar a un entorno virtual. La enfermedad del COVID-19 afectó profundamente la vida en todo el mundo. El aislamiento, las restricciones de contacto y el cierre económico imponen un cambio completo en el entorno psicosocial en los países afectados. Investigaciones científicas (Brooks et al., 2020; Fiorillo \& Gorwood, 2020; Gao et al., 2020; Lima et al., 2020) y organizaciones internacionales (Committee, 2020; World Health Organization, 2020c) han reconocido el impacto psicológico en la población general además de la previamente afectada. La mayoría de los estudios revisados informaron efectos psicológicos negativos, incluidos síntomas de estrés postraumático, confusión y enojo. Los factores estresantes incluyeron la prolongación de la duración de la cuarentena, temores de infección, frustración, aburrimiento, suministros inadecuados, información inadecuada, pérdidas financieras y estigma. 
En el contexto actual de COVID-19, los gobiernos están utilizando varias herramientas para tomar decisiones relacionadas con las políticas públicas, como las aplicaciones móviles para recopilar grandes datos relacionados con los movimientos de los ciudadanos en la ciudad o los patrones de consumo. Un ejemplo de esto es la aplicación "Cuidar" de Argentina, que fue diseñada para rastrear los desplazamientos de las personas cuando solicitan autorización. Aprovechar esa información para diseñar pruebas de BI puede ayudar a los gobiernos a tomar respuestas rápidas y a pivotar frente a resultados inmediatos, facilitando decisiones específicas del contexto frente a demandas urgentes. Por ejemplo, analizar qué áreas o carreteras de cada ciudad se utilizan de forma recurrente en este contexto y su marco temporal para ajustar la sincronización de los mensajes relevantes.

La clave es que las campañas se mantengan, pero se vayan adaptando a las circunstancias cambiantes, a medida que se filtran las buenas y las malas noticias sobre la enfermedad, y cambian las percepciones de riesgo de las personas (Rimal \& Real, 2003). Las autoridades deben mantener un monitoreo constante de los cambios de hábitos en la población, y realizar un rastrillaje sobre las modificaciones en creencias y percepciones que los generan para poder adaptar la comunicación e intervenciones pertinentes a cada contexto.

\section{Conclusión}

En conclusión, el comportamiento humano juega un papel central en la reducción de la propagación del Coronavirus. La comunicación por parte de entidades gubernamentales y otras fuentes confiables sobre los comportamientos deseables y obligatorios durante la pandemia es fundamental. Podemos dar un paso más con la mensajería basada en el comportamiento, relevante al contexto y comunicada a través de nuevos canales de distribución. Esto incluye lograr que los comportamientos deseados sean simples y fáciles de hacer y que los mensajes sean pegadizos y memorables, y apelar a la naturaleza social de las personas al promover la acción colectiva y la cooperación.

Los apoyos sociales y los planes de comportamiento pueden reducir los efectos psicológicos negativos del aislamiento, reduciendo potencialmente el desincentivo para aislar. El comportamiento del espíritu público es más probable con la comunicación frecuente de lo que es "mejor para todos", una fuerte identidad de grupo y la desaprobación social del incumplimiento. La comunicación de crisis eficaz implica rapidez, honestidad, credibilidad, empatía y promover acciones individuales útiles. Es probable que los riesgos se comuniquen mejor a través de números, con rangos para describir la incertidumbre; simplemente indicar un máximo puede sesgar la percepción pública. Los hallazgos apuntan a ser útiles no solo para el gobierno y las autoridades de salud pública, sino también para organizaciones y comunidades. 


\section{Referencias}

Akesson, J., Ashworth-Hayes, S., Hahn, R., Metcalfe, R. D., \& Rasooly, I. (2020). Fatalism, beliefs, and behaviors during the COVID-19 pandemic. National Bureau of Economic Research. https://www.doi.org/10.3386/w2 7245

Ashraf, N. (2013). Rx: Human nature. Harvard business review, 91(4), 119123, 125, 143. PMID: 23593771

Behavioural Insights Team. (2010). Applying behavioural insight to health. London: Cabinet Office. https://assets.publishing.service.g ov.uk/government/uploads/syste $\mathrm{m} /$ uploads/attachment_data/file/6 0524/403936_BehaviouralInsight_ acc.pdf

Behavioural Insights Team. (2013). Applying behavioural insights to charitable giving. Cabinet Office, 1 25. https://good2give.ngo/wpcontent/uploads/2016/04/CAF_Ch aritable_Giving_Report_May_2013.p df

Behavioural Insights Team. (2014). EAST: Four simple ways to apply behavioural insights. Behavioural Insight Team, London. https://www.bi.team/publications /east-four-simple-ways-to-applybehavioural-insights/

Behavioral Insights Team. (2019, October 1). Aprendizajes conductuales para las ciudades. Caracas: Behavioral Insights Team. http://scioteca.caf.com/handle/12 $3456789 / 1470$

Betsch, C., Wieler, L. H., \& Habersaat, K. (2020). Monitoring behavioural insights related to COVID-19. The Lancet, 395(10232), 1255-1256. https://doi.org/10.1016/S01406736(20)30729-7

Bettinger, E. P., Long, B. T., Oreopoulos, P., \& Sanbonmatsu, L. (2012). The role of application assistance and information in college decisions: Results from the H\&R Block FAFSA experiment. The Quarterly Journal of Economics, 127(3), 1205-1242. https://doi.org/10.1093/qje/qjs01 7

Bicchieri, C., \& Dimant, E. (2019). Nudging with care: The risks and benefits of social information. Public choice, 122. https://doi.org/10.1007/s11127019-00684-6

Bowles, J., Larreguy, H., \& Liu, S. (2020). Countering misinformation via WhatsApp: Preliminary evidence from the COVID-19 pandemic in Zimbabwe. PloS one, 15(10), e0240005. https://doi.org/10.1371/journal.p one.0240005

Brockmeyer, A., Kettle, S., \& Smith, S. D. (2016). Casting the tax net wider: 
Experimental evidence from Costa Rica. World Bank policy research working paper, 7850. https://ssrn.com/abstract $=284914$ 2

Brooks, S. K., Webster, R. K., Smith, L. E., Woodland, L., Wessely, S., Greenberg, N., \& Rubin, G. J. (2020). The psychological impact of quarantine and how to reduce it: Rapid review of the evidence. The lancet, 395(10227), 912-920. https://doi.org/10.1016/S01406736(20)30460-8

Cahill, K., \& Perera, R. (2011). Competitions and incentives for smoking cessation. Cochrane Database of Systematic Reviews, 4. https://doi.org/10.1002/1465185 8.CD004307.pub4

Calvo-González, O., \& Zoratto, L. (2017). Behavioral insights for development: Cases from Central America. World Bank Publications. http://hdl.handle.net/10986/2833 5

Cartaud, A., Quesque, F., \& Coello, Y. (2020). Beware of virus! Wearing a face mask against COVID-19 results in a reduction of social distancing. 10.31234/osf.io/ubzea

Castro, L., \& Scartascini, C. (2015). Tax compliance and enforcement in the pampas evidence from a field experiment. Journal of Economic Behavior \& Organization, 116, 6582.

https://publications.iadb.org/publi
cations/english/document/TaxCompliance-and-Enforcement-inthe-Pampas-Evidence-from-aField-Experiment.pdf

Committee, I.-A. S. (2020). Briefing note on addressing mental health and psychosocial aspects of COVID-19 OutbreakVersion 1.1 . https://www.socialserviceworkfor ce.org/resources/briefing-noteaddressing-mental-health-andpsychosocial-aspects-covid-19outbreak-version

Datta, S., Miranda, J. J., Zoratto, L., CalvoGonzález, O., Darling, M., \& Lorenzana, K. (2015). A Behavioral Approach to Water Conservation: Evidence from Costa Rica (SSRN Scholarly Paper ID 2613750). Social Science Research Network. https://papers.ssrn.com/abstract= 2613750

De Ridder, D., Feitsma, J., Van Den Hoven, M., Kroese, F., Schillemans, T., Verweij, M., Venema, T., Vugts, A., \& De vet, E. (2020). Simple nudges that are not so easy. Behavioural Public Policy, 1-19. https://doi.org/doi:10.1017/bpp.2 020.36

Duan, L., \& Zhu, G. (2020). Psychological interventions for people affected by the COVID-19 epidemic. The Lancet Psychiatry, 7(4), 300-302. https://doi.org/10.1016/S22150366(20)30073-0

Edge, J. A., Jakes, R. W., Roy, Y., Hawkins, M., Winter, D., Ford-Adams, M. E., 
Murphy, N. P., Bergomi, A., Widmer, B., \& Dunger, D. B. (2006). The UK case-control study of cerebral oedema complicating diabetic ketoacidosis in children. Diabetologia, 49(9), 2002-2009. https://doi.org/10.1007/s00125006-0363-8

Escandón, K., Rasmussen, A. L., Bogoch, I., Murray, E. J., \& Escandón, K. (2020). COVID-19 and false dichotomies: Time to change the black-or-white messaging about health, economy, SARS-CoV-2 transmission, and masks. BMC Infectious Diseases, 21, 710

https://doi.org/10.1186/s12879021-06357-4

Festinger, L. (1962). A Theory of Cognitive Dissonance. Vol. 2 Stanford University Press. Stanford, CA, USA.

Finset, A., Bosworth, H., Butow, P., Gulbrandsen, P., Hulsman, R. L., Pieterse, A. H., Street, R., Tschoetschel, R., \& van Weert, J. (2020). Effective health communication-a key factor in fighting the COVID-19 pandemic. Patient education and counseling, 103(5), 873. https://dx.doi.org/10.1016\%2Fj.pe c.2020.03.027

Fiorillo, A., \& Gorwood, P. (2020). The consequences of the COVID-19 pandemic on mental health and implications for clinical practice. European Psychiatry, 63(1). https://doi.org/10.1192/j.eurpsy.2 020.35
Fiszbein, A., \& Schady, N. R. (2009). Conditional cash transfers: Reducing present and future poverty. World Bank Publications. http://hdl.handle.net/10986/2597

Galea, S., Merchant, R. M., \& Lurie, N. (2020). The mental health consequences of COVID-19 and physical distancing: The need for prevention and early intervention. JAMA internal medicine, 180(6), 817-818. http://doi.org/10.1001/jamaintern med.2020.1562

Galizzi, M. M. (2017). Behavioral aspects of policy formulation: Experiments, behavioral insights, nudges. En Handbook of policy formulation (pp. 410-430). Edward Elgar Publishing.

Gao, J., Zheng, P., Jia, Y., Chen, H., Mao, Y., Chen, S., Wang, Y., Fu, H., \& Dai, J. (2020). Mental health problems and social media exposure during COVID-19 outbreak. Plos one, 15(4), e0231924.

https://doi.org/10.1371/journal.p one.0231924

García Prado, A. (2016). How to Change Behavior to Improve Maternal and Neonatal Health in Rural Areas of Latin America. Research Departament, Technical Note IDBTN-1071, Inter-American Development Bank, Washington DC. https://publications.iadb.org/en/p ublication/17202/how-changebehavior-improve-maternal-andneonatal-health-rural-areas-latin 
García Zaballos, A., Iglesias, E., Prado, G., Árias, G., Huici, H., Puig, P., Martínezgarza, R., \& Cabello, S. (2020). Digitalización: Herramienta de defensa ante la crisis del Covid-19 y para el desarrollo sostenible. InterAmerican Development Bank. https://doi.org/10.18235/000235

6

Garfin, D. R., Silver, R. C., \& Holman, E. A. (2020). The novel coronavirus (COVID-2019) outbreak: Amplification of public health consequences by media exposure. Health psychology. https://psycnet.apa.org/doi/10.10 37/hea0000875

Greenberg, N., Docherty, M., Gnanapragasam, S., \& Wessely, S. (2020). Managing mental health challenges faced by healthcare workers during covid-19 pandemic. bmj, 368. https://doi.org/10.1136/bmj.m12 11

Hallsworth, M., \& Kirkman, E. (2020). Behavioral insights. MIT Press.

Hallsworth, M., Snijders, V., Burd, H., Prestt, J., Judah, G., Huf, S., \& Halpern, D. (2016). Applying behavioral insights: Simple ways to improve health outcomes. World Innovation Summit for Health, Doha, Qatar, 2930

November. https://www.bi.team/publications /applying-behavioural-insightssimple-ways-to-improve-healthoutcomes/
Halpern, D. (2015). Inside the nudge unit: How small changes can make a big difference. Random House.

Hossain, M. M., Sultana, A., \& Purohit, N. (2020). Mental health outcomes of quarantine and isolation for infection prevention: A systematic umbrella review of the global evidence. Available at SSRN 3561265.

https://dx.doi.org/10.4178\%2Fepi h.e2020038

Ienca, M., \& Vayena, E. (2020). On the responsible use of digital data to tackle the COVID-19 pandemic. Nature medicine, 26(4), 463-464. https://doi.org/10.1038/s41591020-0832-5

Imhoff, R., \& Lamberty, P. (2020). A bioweapon or a hoax? The link between distinct conspiracy beliefs about the Coronavirus disease (COVID-19) outbreak and pandemic behavior. Social Psychological and Personality Science, 11(8), 11101118.

https://doi.org/10.1177\%2F19485 50620934692

John, L. K., Loewenstein, G., Troxel, A. B., Norton, L., Fassbender, J. E., \& Volpp, K. G. (2011). Financial incentives for extended weight loss: A randomized, controlled trial. Journal of general internal medicine, 26(6), 621-626. https://doi.org/10.1007/s11606010-1628-y 
Jordan, J., Yoeli, E., \& Rand, D. (2020). Don't get it or don't spread it? Comparing self-interested versus prosocially framed COVID-19 prevention messaging. https://doi.org/10.31234/osf.io/y uq7 $\mathrm{x}$

Kahneman, D., \& Egan, P. (2011). Thinking, fast and slow. vol. 1 Farrar. Straus and Giroux New York.

Kaslow, N. J., Friis-Healy, E. A., Cattie, J. E., Cook, S. C., Crowell, A. L., Cullum, K. A., Del Rio, C., Marshall-Lee, E. D., LoPilato, A. M., \& VanderBroekStice, L. (2020). Flattening the emotional distress curve: A behavioral health pandemic response strategy for COVID-19. American Psychologist. https://psycnet.apa.org/doi/10.10 37/amp0000694

Kessler, R. C., \& Wittchen, H.-U. (2008). Post-disaster mental health need assessment surveys-The challenge of improved future research. International Journal of Methods in Psychiatric Research, 17 Suppl 2, S15.

https://doi.org/10.1002/mpr.266

Kettle, S., Hernandez, M., Ruda, S., \& Sanders, M. (2016). Behavioral interventions in tax compliance: Evidence from Guatemala. The World Bank. https://doi.org/10.1596/18139450-7690

King, D. L., Haagsma, M. C., Delfabbro, P. H., Gradisar, M., \& Griffiths, M. D.
(2013). Toward a consensus definition of pathological videogaming: A systematic review of psychometric assessment tools. Clinical psychology review, 33(3), 331-342.

https://doi.org/10.1016/j.cpr.201 3.01 .002

Klingensmith, G. J., Aisenberg, J., Kaufman, F., Halvorson, M., Cruz, E., Riordan, M. E., Varma, C., Pardo, S., Viggiani, M. T., \& Wallace, J. F. (2013). Evaluation of a combined blood glucose monitoring and gaming system (Didget $\circledast$ ) for motivation in children, adolescents, and young adults with type 1 diabetes. Pediatric diabetes, 14(5), 350-357. https://doi.org/10.1111/j.13995448.2011.00791.x

Kuper-Smith, B. J., Doppelhofer, L. M., Oganian, Y., Rosenblau, G., \& Korn, C. (2020). Optimistic beliefs about the personal impact of COVID-19. https://doi.org/10.31234/osf.io/e pcyb

Larkin, C., Sanders, M., Andresen, I., \& Algate, F. (2018). Testing local descriptive norms and salience of enforcement action: A field experiment to increase tax collection. Available at SSRN 3167575. https://dx.doi.org/10.2139/ssrn.3 167575

Lewandowsky, S., Ecker, U. K., Seifert, C. M., Schwarz, N., \& Cook, J. (2012). Misinformation and its correction: Continued influence and successful 
debiasing. Psychological science in the public interest, 13(3), 106-131. https://doi.org/10.1177\%2F15291 00612451018

Lima, C. K. T., de Medeiros Carvalho, P. M., Lima, I. de A. A. S., de Oliveira Nunes, J. V. A., Saraiva, J. S., de Souza, R. I., da Silva, C. G. L., \& Neto, M. L. R. (2020). The emotional impact of Coronavirus 2019-nCoV (new Coronavirus disease). Psychiatry research, 287, 112915. https://doi.org/10.1016/j.psychre s.2020.112915

Liu, P. L. (2020). COVID-19 information seeking on digital media and preventive behaviors: The mediation role of worry. Cyberpsychology, Behavior, and Social Networking, 23(10), 677-682. https://doi.org/10.1089/cyber.202 0.0250

Lourenço, J. S., Ciriolo, E., Almeida, S. R., \& Troussard, X. (2016). Behavioural insights applied to policy: European Report 2016. Brussels: European Union.

Lunn, P. D., Belton, C. A., Lavin, C., McGowan, F. P., Timmons, S., \& Robertson, D. A. (2020). Using Behavioral Science to help fight the Coronavirus. Journal of Behavioral Public Administration, $3(1)$.

http://hdl.handle.net/10419/2379 28

Lunn, P. D., Timmons, S., Belton, C. A., Barjaková, M., Julienne, H., \& Lavin, C. (2020). Motivating social distancing during the Covid-19 pandemic: An online experiment. Social Science \& Medicine, 265, 113478.

https://doi.org/10.1016/j.socscim ed.2020.113478

Lunn, P. D., Timmons, S., Julienne, H., Belton, C. A., Barjaková, M., Lavin, C., \& McGowan, F. P. (2020). Using decision aids to support selfisolation during the COVID-19 pandemic. Psychology \& Health, 119.

https://doi.org/10.1080/0887044 6.2020 .1849701

Madrian, B. C. (2014). Applying insights from behavioral economics to policy design. Annu. Rev. Econ., 6(1), 663-688.

Martínez Villareal, D., Méndez, A. M. R., \& Scartascini, C. (2020). La economía del comportamiento puede ayudar a combatir el coronavirus. Banco Interamericano del Desarrollo. http://dx.doi.org/10.18235/00023 15

Mayer, S. E., Kalil, A., Oreopoulos, P., \& Gallegos, S. (2015). Using behavioral insights to increase parental engagement: The parents and children together (PACT) intervention. National Bureau of Economic Research. https://doi.org/10.3386/w21602

OECD. (2017a). Behavioural insights and public policy. Lessons from Around the World, OECD Publishing, Paris, 
https://doi.org/10.1787/9789264 270480-en.

OECD. (2017b). Use of Behavioural Insights in Consumer Policy. OECD Paris. https://doi.org/10.1787/c2203c35 -en

OECD. (2019). Delivering Better Policies Through Behavioural Insights: New Approaches. OECD Publishing, Paris, https://doi.org/10.1787/6c9291e2 -en

OECD. (2020). Tax Policy Reforms 2020: OECD and Selected Partner Economies (OECD Publishing). OECD.

https://doi.org/10.1787/7af51916 -en

Olson, O., Berry, C., \& Kumar, N. (2020). Addressing Parental Vaccine Hesitancy towards Childhood Vaccines in the United States: A Systematic Literature Review of Communication Interventions and Strategies. Vaccines, 8(4), 590. https://doi.org/10.3390/vaccines8 040590

Pennycook, G., McPhetres, J., Zhang, Y., Lu, J. G., \& Rand, D. G. (2020). Fighting COVID-19 Misinformation on Social Media: Experimental Evidence for a Scalable Accuracy-Nudge Intervention. Psychological Science, 31(7), 770-780. https://doi.org/10.1177/0956797 620939054

Rimal, R. N., \& Real, K. (2003). Perceived risk and efficacy beliefs as motivators of change: Use of the risk perception attitude (RPA) framework to understand health behaviors. Human communication research, 29(3), 370-399. https://doi.org/10.1111/j.14682958.2003.tb00844.x

Rimal, R. N., \& Storey, J. D. (2020). Construction of Meaning during a Pandemic: The Forgotten Role of Social Norms. Health communication, 35(14), 1732-1734. https://doi.org/10.1080/1041023 6.2020 .1838091

Rudd, M., Aaker, J., \& Norton, M. I. (2014). Getting the most out of giving: Concretely framing a prosocial goal maximizes happiness. Journal of Experimental Social Psychology, 54, 11-24

https://doi.org/10.1016/j.jesp.201 4.04 .002

Sanders, M. (2017). Social influences on charitable giving in the workplace. Journal of behavioral and experimental economics, 66, 129136.

https://doi.org/10.1016/j.socec.20 15.12 .004

Sanketh Andhavarapu. (2020, diciembre 7). A Behavioral Approach to COVID-19 Vaccine Hesitancy. The Decision Lab. https://thedecisionlab.com/insight s/health/a-behavioral-approachto-covid-19-vaccine-hesitancy/

Soper, G. A. (1919). The lessons of the pandemic. Science, 49(1274), 501506. 
https://doi.org/10.1126/science.4 9.1274.501

Sunstein, C. R., Reisch, L. A., \& Rauber, J. (2017). Behavioral insights all over the world? Public attitudes toward nudging in a multi-country study. Public Attitudes Toward Nudging in a Multi-Country Study (February 21, 2017).

https://dx.doi.org/10.2139/ssrn.2 921217

Surrey, S. S. (1970). Tax incentives as a device for implementing government policy: A comparison with direct government expenditures. Harvard Law Review, 705-738.

https://doi.org/10.2307/1339837

Thaler, R. H., \& Sunstein, C. R. (2009). Nudge: Improving decisions about health, wealth, and happiness. Penguin.

Tversky, A., \& Kahneman, D. (1974). Judgment under uncertainty: Heuristics and biases. science, 185(4157), 1124-1131. https://doi.org/10.1126/science.1 85.4157.1124

Urzúa, A., Vera-Villarroel, P., Caqueo-Urízar, A., \& Polanco-Carrasco, R. (2020). La Psicología en la prevención y manejo del COVID-19. Aportes desde la evidencia inicial. Terapia psicológica, $\quad 38(1), \quad$ 103-118. http://dx.doi.org/10.4067/S071848082020000100103

Usher, K., Bhullar, N., \& Jackson, D. (2020). Life in the pandemic: Social isolation and mental health. Wiley Online Library. https://doi.org/10.1111/jocn.1529 0

Van Bavel, J. J., Baicker, K., Boggio, P. S., Capraro, V., Cichocka, A., Cikara, M., Crockett, M. J., Crum, A. J., Douglas, K. M., \& Druckman, J. N. (2020). Using social and behavioural science to support COVID-19 pandemic response. Nature human behaviour, 4(5), 460-471. https://doi.org/10.1038/s41562020-0884-z

Vlaev, I., King, D., Dolan, P., \& Darzi, A. (2016). The theory and practice of "nudging": Changing health behaviors. Public Administration Review, 76(4), 550-561. https://doi.org/10.1111/puar.125 64

Volpp, K. G., Troxel, A. B., Pauly, M. V., Glick, H. A., Puig, A., Asch, D. A., Galvin, R., Zhu, J., Wan, F., \& DeGuzman, J. (2009). A randomized, controlled trial of financial incentives for smoking cessation. $N$ Engl $\mathrm{J} \mathrm{Med,}$ 360 , 699-709. https://doi.org/10.1056/NEJMsa0 806819

West, R., Michie, S., Rubin, G. J., \& Amlôt, R. (2020). Applying principles of behaviour change to reduce SARSCoV-2 transmission. Nature Human Behaviour, 4(5), 451-459. https://doi.org/10.1038/s41562020-0887-9 
Whitelaw, S., Mamas, M. A., Topol, E., \& Van Spall, H. G. (2020). Applications of digital technology in COVID-19 pandemic planning and response. The Lancet Digital Health. https://doi.org/10.1016/S25897500(20)30142-4

World Health Organization. (2020a). Advice on the use of masks in the context of COVID-19: Interim guidance, 5 June 2020. World Health Organization. World Health Organization. https://apps.who.int/iris/handle/ $10665 / 332293$.

World Health Organization. (2020b). Behavioural considerations for acceptance and uptake of COVID-19 vaccines: WHO technical advisory group on behavioural insights and sciences for health, meeting report, 15 October 2020.
World Health Organization. (2020c). Mental health and psychosocial considerations during the COVID19 outbreak, 18 March 2020. World Health Organization. https://apps.who.int/iris/handle/ $10665 / 331490$

World Health Organization. (2020d). Survey tool and guidance: Rapid, simple, flexible behavioural insights on COVID-19: 29 July 2020. World Health Organization. Regional Office for

Europe. https://apps.who.int/iris/handle/ $10665 / 333549$. 